\title{
The Semantics of Rational Contractions
}

Jürgen Giesl

Fachbereich Informatik

Technische Hochschule Darmstadt

Alexanderstr. 10

64283 Darmstadt

Tel.: ++49-6151-166658

Fax: ++49-6151-165326

E-mail: giesl@inferenzsysteme.informatik.th-darmstadt.de
Ingrid Neumann

Fakultät für Informatik

Universität Karlsruhe

Kaiserstr. 12

76128 Karlsruhe

Tel.: ++49-721-6084375

E-mail: neumann@ira.uka.de

\begin{abstract}
This paper is concerned with the revision of beliefs in the face of new and possibly contradicting information. In the Logic of Theory Change developed by Alchourron, Gärdenfors and Makinson this nonmonotonic process consists of a contraction and an expansion of a set of formulas. To achieve minimal change they formulated widely accepted postulates that rational contractions have to fulfill.

Contractions as defined by Alchourron, Gärdenfors and Makinson only operate on deductively closed sets of formulas. Therefore they cannot be used in practical applications, eg. knowledge representation, where only finitely representable sets can be handled.

We present a semantical characterization of rational finite contractions (the class of rational contractions maintaining finite representability) which provides an insight into the true nature of these operations. This characterization shows all possibilities to define concrete functions possessing these properties.

When regarding concrete contractions known from literature in the light of our characterization we have found that they are all defined according to the same semantical strategy of minimal semantical change. As this strategy does not correspond to the goal of keeping as many important formulas as possible in the contracted set, we suggest a finite contraction defined according to the new strategy of maximal maintenance.
\end{abstract}

\section{Keywords}

Nonmonotonic Logic, Logic of Theory Change, Knowledge Representation 


\section{Introduction}

We regard the classical propositional calculus. Let $S$ be a signature, i.e. a countable non-empty set of propositional variables. Then For $_{S}$ denotes the set of propositional formulas built with symbols from $S$ and logical connectives. For $A \subseteq$ For $_{S}$ we define $\mathrm{Cn}_{S}(A)=\left\{x \in \mathrm{For}_{S} \mid A \vDash x\right\}^{1}$. The set of theories on $S$ is $\mathcal{T}(S)=\{A$ $\subseteq$ For $\left._{S} \mid A=\mathrm{Cn}_{S}(A)\right\}$, i.e. the set of all deductively closed sets of formulas.

The expansion of a theory $A$ by a formula $x$, written as $A+x$, means the simple addition of that formula under preservation of the deductive closure, i.e. $A+x=$ $\mathrm{Cn}_{S}(A \cup\{x\})$. The revision of a theory $A$ by a formula $x$, written as $A * x$, means the expansion of $A$ by $x$ satisfying the condition that $x$ is an element of $A * x$ and $A * x$ is consistent (under the assumption that $\neg x$ is not a tautology). The contraction of a theory $A$ by a formula $x$, written $A-x$, is the removal of $x$ from $A$ resulting again in a deductively closed set. Revisions satisfying the rationality postulates can be defined in terms of contractions if we make use of the Levi identity $A * x=$ $(A-\neg x)+x$. Thus we only take contractions into account.

After identifying these three classes of operations Alchourron, Gärdenfors and Makinson $^{2}$ have formulated the following postulates determining the rationality of contractions.

\section{Definition 1}

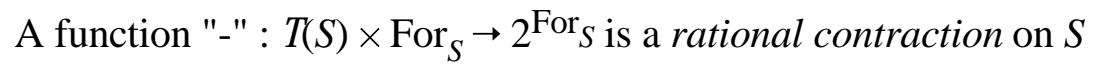

iff

for every $A \in \mathcal{T}(S)$ and for every $\{x, y\} \subseteq$ For $_{S}$,

1. $A-x \in \mathcal{T}(S)$

2. $A-x \subseteq A$

3. if $x \notin A$ then $A-x=A$

4. if $\not \neq x$ then $x \notin A-x$

5. if $x \in A$ then $A \subseteq \mathrm{Cn}_{S}((A-x) \cup\{x\})$

6. if $\vDash x \leftrightarrow y$ then $A-x=A-y$

"-" is a fully rational contraction on $S$

iff

"-" is a rational contraction on $S$

and for every $A \in \mathcal{T}(S)$ and for every $\{x, y\} \subseteq$ For $_{S}$,

7. $A-x \cap A-y \subseteq A-(x \wedge y)$

8. if $x \notin A-(x \wedge y)$ then $A-(x \wedge y) \subseteq A-x$

\footnotetext{
$1 " \models "$ denotes logical consequence.

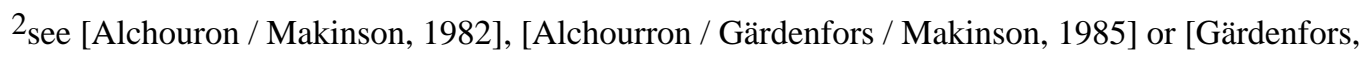
1988].
} 
In practical applications a theory $A$ has to be representable in a finite way, eg. by a finite base $B$ such that $A=\mathrm{Cn}_{S}(B)$. Let $\mathcal{T}_{\mathrm{F}}(S)$ denote the set of all theories on $S$ possessing a finite base. Then our main interest is in those rational contractions which transform each $A \in \mathcal{T}_{\mathrm{F}}(S)$ into another finitely representable theory $A-x \in \mathcal{T}_{\mathrm{F}}(S)$.

\section{Definition 2}

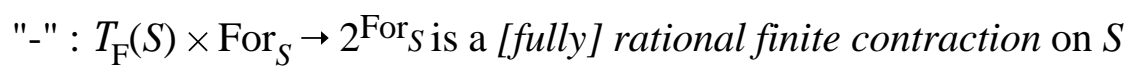

iff

"-" can be extended to a [fully] rational contraction on $S$

and for every $A \in \mathcal{T}_{\mathrm{F}}(S)$ and for every $x \in$ For $_{S}, A-x \in \mathcal{T}_{\mathrm{F}}(S)$.

Certain functions of that kind have already been published in literature ${ }^{3}$. But the whole range of possibilities to define such an operation has not yet been evident. As a real comprehension of this class of functions is only possible on a semantical level we develop a semantical characterization of rational finite contractions in section 2 . By means of this result we present concrete finite contractions in section 3, investigate their relationship and suggest a new strategy capturing the intuitive notion of minimal change.

\section{Semantical Characterization of Rational Contractions}

First we show what the existence of a finite base for a theory means semantically. Next the notion of relevant atoms is introduced and finally we present the semantical characterization of rational finite contractions.

Let $\operatorname{Int}(S)$ denote the set of all interpretations ${ }^{4}$ on $S$ and let $R$ be a non-empty subset of $S$. Then we define two transformation functions $\mathrm{t}^{+}{ }_{R, S}: 2^{\operatorname{Int}(R)} \rightarrow 2^{\operatorname{Int}(S)}$ and $\mathrm{t}^{-}{ }_{S, R}: 2^{\operatorname{Int}(S)} \rightarrow 2^{\operatorname{Int}(R)}$, such that for every $\mathfrak{I}^{\prime} \subseteq \operatorname{Int}(R), \mathrm{t}^{+}{ }_{R, S}\left(\mathfrak{I}^{\prime}\right)=\{I \in$ $\left.\operatorname{Int}(S)|I|_{R} \in \mathfrak{I}^{\prime}\right\}$ and for every $\mathfrak{I} \subseteq \operatorname{Int}(S), \mathrm{t}^{-}, R(\mathfrak{I})=\left\{\left.I\right|_{R} \mid I \in \mathfrak{I}\right\}$, where $\left.I\right|_{R}$ denotes the restriction of $I$ to $R$. Thus $\mathrm{t}^{+}{ }_{R, S}$ transforms sets of interpretations on $R$ into the corresponding ones on $S$ and $\mathrm{t}^{-}{ }_{S, R}$ works vice versa.

For every set of interpretations $\mathfrak{I} \subseteq \operatorname{Int}(S), \mathfrak{I} \subseteq \mathrm{t}^{+}{ }_{R, S}\left(\mathrm{t}^{-}{ }_{S, R}(\mathfrak{I})\right)$ is obviously true. We call $\mathfrak{I}$ reducible to $R$ iff $\mathfrak{I}=\mathrm{t}^{+}{ }_{R, S}\left(\mathrm{t}^{-}{ }_{S, R}(\mathfrak{I})\right)$ holds as well, i.e. if $\mathfrak{I}$ is uniquely determined by a set of interpretations on $R$.

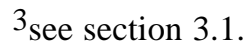

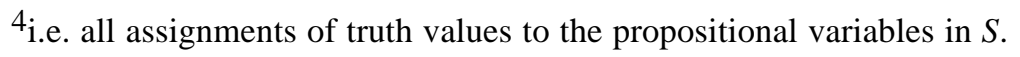




\section{Example 1}

Let $S=\{a, b, c\}, A=\mathrm{Cn}_{S}(\{a, b\})$. Then $\operatorname{Mod}_{S}(A)$ contains all interpretations mapping $a$ and $b$ to true. $\mathrm{t}_{S,\{a, c\}}^{-}\left(\operatorname{Mod}_{S}(A)\right)$ consists of the two interpretations on $\{a, c\}$ mapping $a$ to true. Therefore $\mathrm{t}^{+}\{a, c\}, S\left(\mathrm{t}_{S,\{a, c\}}\left(\operatorname{Mod}_{S}(A)\right)\right)$ contains all interpretations on $S$ that map a to true. So $\operatorname{Mod}_{S}(A)$ is not reducible to $\{a, c\}$.

If $A \subseteq$ For $_{S}$ is a set of formulas then $\operatorname{Mod}_{S}(A)$ denotes the set of all its models ${ }^{5}$ on $S$. The following lemma ${ }^{6}$ characterizes theories possessing finite bases by stating that these theories can be represented by interpretations on finite subsignatures.

\section{Lemma 1}

Let $A \in \mathcal{T}(S)$ be a theory.

$A$ possesses a finite base, i.e. $A \in \mathcal{T}_{\mathrm{F}}(S)$

iff

$\operatorname{Mod}_{S}(A)$ is reducible to some finite non-empty subset of $S$.

\section{Example 2}

Let $\mathfrak{I}$ consist of all interpretations $I$ on $S$ such that $\{a \in S \mid I(a)=$ false $\}$ is finite. Then $\mathfrak{I}$ is reducible to some subsignature $R \subseteq S$ iff $S \backslash R$ is finite. Therefore $\mathfrak{I}$ is not reducible to any finite subsignature if $S$ is infinite.

The next definition is essential for the following characterization theorem as well as for the definition of concrete contractions to be presented in the next section.

\section{Definition 3}

Let $\mathfrak{I} \subseteq \operatorname{Int}(S)$ be a set of interpretations on $S$ and let $\$$ be the set of all non-empty subsets of $S$ that $\mathfrak{I}$ is reducible to. Then the relevant atoms of $\mathfrak{I}$ are defined as

$$
\operatorname{rat}(\mathfrak{I})=\left\{\begin{array}{l}
\bigcap R, \text { if } \mathfrak{I} \neq \varnothing \text { and } \mathfrak{I} \neq \operatorname{Int}(S) \\
R \in \$ \\
\varnothing, \text { otherwise. }
\end{array}\right.
$$

\section{Example 1 (cont.)}

$\operatorname{rat}\left(\operatorname{Mod}_{S}(A)\right)=\{a, b\} \cap\{a, b, c\}=\{a, b\}$.

\section{Example 2 (cont.)}

$\operatorname{rat}(\mathfrak{I})=\bigcap \quad R=\varnothing$.

$$
S \backslash R \text { finite }
$$

$5_{\text {i.e. all interpretations evaluating every } x \in A \text { to true. }}$
${ }^{6}$ All proofs can be found in [Giesl, 1992]. 
Relevant atoms have the following important property.

\section{Lemma 2}

Let $\mathfrak{I} \subseteq \operatorname{Int}(S)$ be a set of interpretations on $S$ such that $\mathfrak{I}$ is reducible to some finite non-empty subsignature and let $R$ be a non-empty subset of $S$.

Then $\mathfrak{I}$ is reducible to $R$

iff

$\operatorname{rat}(\mathfrak{I}) \subseteq R$.

For an $\mathfrak{I}$ meeting the prerequisites of lemma $2 \operatorname{rat}(\mathfrak{I})$ is the smallest subsignature it is reducible to. Note that this were not necessarily true if $\mathfrak{I}$ could not be reduced to any finite subsignature.

\section{Example 1 (cont.)}

$\operatorname{Mod}_{S}(A)$ is reducible to every superset of $\{a, b\}$.

\section{Example 2 (cont.)}

If $S$ is infinite then $\mathfrak{I}$ does not satisfy the prerequisites of lemma 2 . If $S$ is finite $\mathfrak{I}$ is reducible to every subsignature of $S$.

With the aid of these two lemmas it is now possible to characterize the class of rational finite contractions semantically.

\section{Theorem 1}

A function "-" : $\mathcal{T}_{\mathrm{F}}(S) \times$ For $_{S} \rightarrow 2$ For $_{S}$ is a rational finite contraction on $S$ iff

there are functions $j: \mathcal{T}_{\mathrm{F}}(S) \times \operatorname{For}_{S} \rightarrow 2^{\operatorname{Int}(S)}$ and $r: \mathcal{T}_{\mathrm{F}}(S) \times$ For $_{S} \rightarrow 2^{S}$, such that for every $A \in \mathcal{T}_{\mathrm{F}}(S)$ and for every $\{x, y\} \subseteq \operatorname{For}_{S}$, $A-x \in \mathcal{T}(S)$ and $\operatorname{Mod}_{S}(A-x)=\left\{\begin{array}{l}\operatorname{Mod}_{S}(A) \cup \mathrm{t}^{+}{ }_{r(A, x), S}\left(\mathrm{t}^{-}, r(A, x)\right. \\ \\ \operatorname{Mod}_{S}(A), \text { otherwise, }\end{array}\right.$

where $j(A, x) \subseteq \operatorname{Mod}_{S}(\{\neg x\})$, $j(A, x) \neq \varnothing$, if $\not \neq x$, $j(A, x)=j(A, y)$, if $\vDash x \leftrightarrow y$

and $r(A, x)$ is finite and non-empty,

$$
\begin{aligned}
& r(A, x) \supseteq \operatorname{rat}\left(\operatorname{Mod}_{S}(\{x\})\right) \\
& r(A, x)=r(A, y), \text { if } \vDash x \leftrightarrow y .
\end{aligned}
$$


So a rational finite contraction of $A$ by $x$ is an extension of the original set of models $\operatorname{Mod}_{S}(A)$ by some additional interpretations $\mathrm{t}_{r(A, x), S}^{+}\left(\mathrm{t}^{-}{ }_{S, r(A, x)}(j(A, x))\right)$ $\subseteq \operatorname{Mod}_{S}(\{\neg x\})$. This set is reducible to a finite subsignature $r(A, x)$ containing the relevant atoms of $\operatorname{Mod}_{S}(\{x\})$.

Every choice of functions $j$ and $r$ meeting the requirements mentioned above defines a rational finite contraction. (As the following example illustrates this were not always true if $r(A, x)$ were allowed to be infinite, because there exist $\mathfrak{I} \subseteq \operatorname{Int}(S)$ not constituting the set of models of any theory.)

\section{Example 3}

Let $S=\left\{b, a_{0}, a_{1}, a_{2}, \ldots\right\}$ be an infinite signature, $A=\mathrm{Cn}_{S}(\{b\})$. When contracting $A$ by $b$ we let $j(A, b)$ consist of all models of $\neg b$ except the one mapping every atom to false and we let $r(A, b)$ be an infinite signature, namely $r(A, b)=$ $S$. By the above theorem $\operatorname{Mod}_{S}(A-x)$ contains all interpretations on $S$ except the one mapping all atoms to false. But due to the compactness theorem there exists no theory with this set of models.

A corresponding characterization of the class of all rational contractions (not necessarily preserving finite representability) can be obtained when $\mathrm{t}_{r(A, x), S}\left(\mathrm{t}^{-}{ }_{S, r(A, x)}(\right.$ $j(A, x)))$ is replaced by $\operatorname{Mod}_{S}\left(\left\{z \in \operatorname{For}_{S} \mid j(A, x) \subseteq \operatorname{Mod}_{S}(\{z\})\right\}\right)$. Then every function $j$ with properties as demanded in the theorem generates a rational contraction. Therefore this is a stronger result than all former semantical characterizations of rational contractions, eg. those by [Grove, 1988] or [Katsuno / Mendelzon, 1989].

By regarding functions $j$ that select maximal models of $\{\neg x\}$ according to some reflexive and transitive relation on $\operatorname{Int}(S)$ similar characterizations of fully rational contractions as well as of fully rational finite contractions can be formulated.

In the next section we will use our result to define and examine several concrete contractions.

\section{Semantically defined Contractions}

In this section concrete rational finite contractions are defined. We will make use of the semantical characterization presented in the last section which states that determining a certain contraction means choosing two functions $j$ and $r$ possessing the properties described above. 
Strictly speaking we proceed by defining a function $j$ meeting the three prerequisites mentioned in theorem 1 . Then we show that for every $A \in \mathcal{T}_{\mathrm{F}}(S)$ and for every formula $x, j(A, x)$ is reducible to some finite non-empty subsignature $r(A, x) \supseteq$ $\operatorname{rat}\left(\operatorname{Mod}_{S}(\{x\})\right)$ which is only dependent on $\operatorname{Mod}_{S}(A)$ and $\operatorname{Mod}_{S}(\{x\})$. Due to the theorem the extension of $\operatorname{Mod}_{S}(A)$ by such a $j(A, x)$ is a rational finite contraction and every function of this class can be defined that way.

As mentioned in the beginning our aim is to achieve minimal change of $A$ when contracting $A$ by $x$. Therefore $j(A, x)$ should contain those models of $\{\neg x\}$ which are "nearest" to the original models in $\operatorname{Mod}_{S}(A)$. The following contractions only differ in their interpretation of the word "nearest".

We have found that all concrete contractions defined in literature interpret "being near" in a similar way. Their strategy of minimal semantical change is presented in section 3.1 .

But as this semantical strategy does not lead to the desired syntactical consequences we propose a different interpretation of "being near" in section 3.2, namely the strategy of maximal maintenance.

\subsection{Minimal Semantical Change}

The idea of this strategy is to regard those interpretations as "nearest" to a set of interpretations $\mathfrak{I} \subseteq \operatorname{Int}(S)$ that can be obtained by extending $\mathfrak{I}$ as "little" as possible. Of course the ambiguity of the term "little" gives rise to several different contractions as will be demonstrated.

We extend a set of interpretations by generalizing it with respect to some subsignature.

\section{Definition 4}

Let $\mathfrak{I} \subseteq \operatorname{Int}(S)$ be a set of interpretations and let $R$ be a proper subset of $S$.

Then the generalization of $\mathfrak{I}$ with respect to $R$ is defined

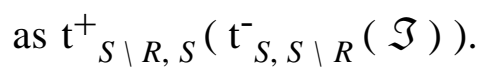

The generalization of $\mathfrak{I}$ with respect to $S$ is defined as $\operatorname{Int}(S)$, if $\mathfrak{I} \neq \varnothing$,

and as $\emptyset$, otherwise.

So the generalization of $\mathfrak{I}$ with respect to $R$ consists of all those $I_{0} \in \operatorname{Int}(S)$ such that there is an $I_{1} \in \mathfrak{I}$ from which they differ on $R$ only. Note that $a \in S$ is a relevant atom of $\mathfrak{I}$ iff the generalization of $\mathfrak{I}$ with respect to $\{a\}$ yields a proper superset of $\mathfrak{I}$. 


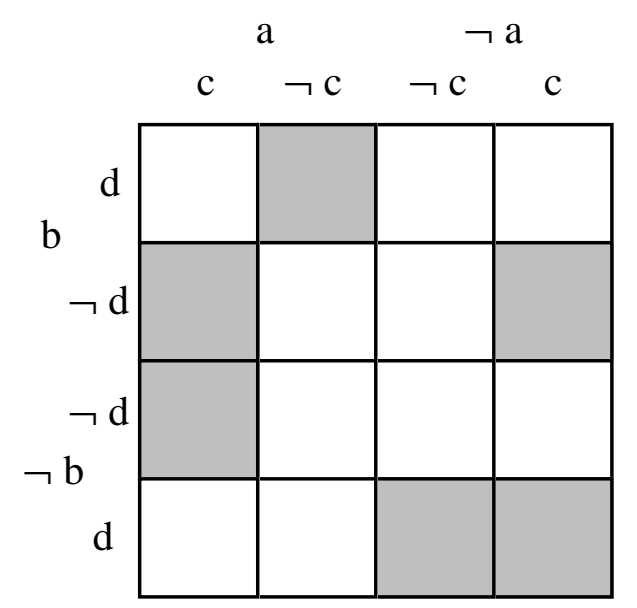

Figure 1: $K V$-diagram for $\operatorname{Mod}_{S}(A)$, Example 4.

\section{Example 4}

We define $S=\{a, b, c, d\}$ and $A=\mathrm{Cn}_{S}(\{c \vee d, \neg a \vee \neg c \vee \neg d, \neg a \vee b \vee$ $\neg d, a \vee \neg b \vee \neg d, a \vee b \vee d\}$ ). This is illustrated by the KV-diagram in Figure 1, where $\operatorname{Mod}_{S}(A)$ is marked grey. Figure 2 shows the set of interpretations resulting from the generalization of $\operatorname{Mod}_{S}(A)$ with respect to $\{a\}$.

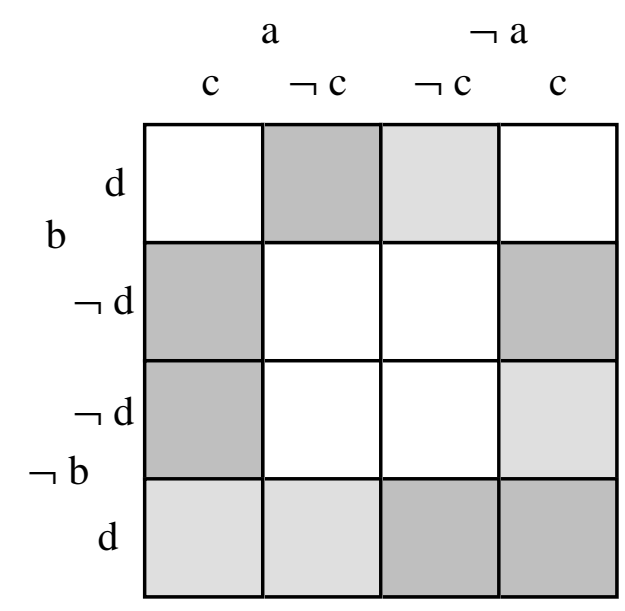

Figure 2: Generalization of $\operatorname{Mod}_{S}(A)$ with respect to $\{a\}$.

We are going to define $j(A, x)$ as the intersection of $\operatorname{Mod}_{S}(\{\neg x\})$ and some $\mathfrak{I} \subseteq$ $\operatorname{Int}(S)$ obtained by generalization. Still there are three decisions to make:

1. Due to the strategy of minimal semantical change we are interested in minimal sets $R$ such that the generalization with respect to $R$ contains models of $\{\neg x\}$. Hence we have to decide between measuring sets by their $\underline{\boldsymbol{c}}$ ardinality or by set $\underline{\boldsymbol{i}} n \mathrm{nclusion}$. 
2. We may determine minimal subsignatures for the generalization of the whole set of models $\operatorname{Mod}_{S}(A)$ or for each $I \in \operatorname{Mod}_{S}(A)$ seperately.

3. We can either generalize with respect to $\underline{e} a c h$ minimal $R$ and take the union of the resulting sets or generalize with respect to the $\underline{\boldsymbol{u}}$ nion of all minimal sets $R$.

As there are $2^{3}=8$ possibilities for the choice of that $\mathfrak{I}$, we obtain eight different functions $\mathrm{j}_{\mathrm{cwe}}, \mathrm{j}_{\mathrm{iwe}}, \ldots, \mathrm{j}_{\mathrm{isu}}$. The name of each function corresponds to the decisions made in its definition.

As a sort of upper bound we define a ninth function $\mathrm{j}_{\mathrm{ra}}$ consisting of those models of $\{\neg x\}$ that can be obtained by generalizing $\operatorname{Mod}_{S}(A)$ with respect to the relevant atoms of $\operatorname{Mod}_{S}(\{x\})^{7}$.

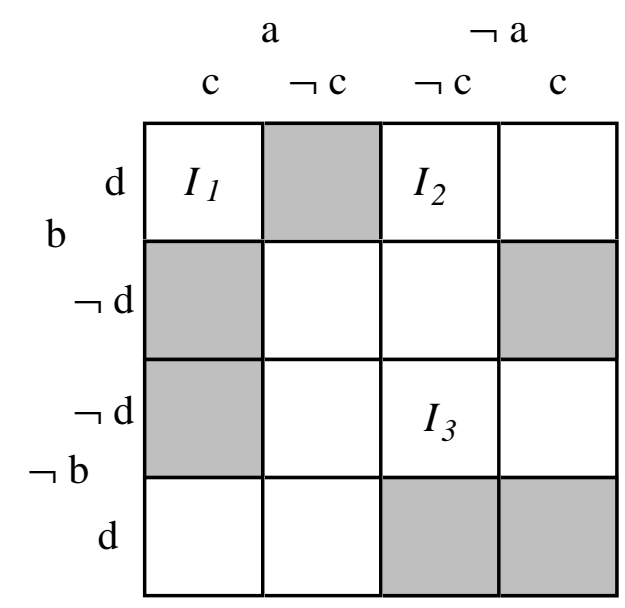

Figure 3: Contracting A by $x$.

\section{Example 4 (cont.)}

When contracting $A$ by $x=\neg(a \leftrightarrow c) \vee \neg(b \leftrightarrow d) \vee(a \wedge \neg b)$, we extend $\operatorname{Mod}_{S}(A)$ by those interpretations from $\operatorname{Mod}_{S}(\{\neg x\})=\left\{I_{1}, I_{2}, I_{3}\right\}$ that can be obtained by generalizations with respect to minimal subsignatures. This is illustrated by figure 3. Generalization with respect to $\{a\}$ or $\{b\}$ yields $I_{2}$, while generalization with respect to $\{c\}$ leads to $I_{1}$ and generalization with respect to $\{d\}$ results in $I_{1}$ and $I_{3}$. Therefore $\mathrm{j}_{\mathrm{cwe}}(A, x)=\ldots=\mathrm{j}_{\mathrm{ra}}(A, x)=\left\{I_{1}, I_{2}, I_{3}\right\}$.

\footnotetext{
${ }^{7}$ To constitute a rational contraction $j(A, x)$ has to be non-empty if $x$ is not a tautology. Therefore we define $\mathrm{j}_{\mathrm{c} w \mathrm{e}}\left(\right.$ For $\left._{S}, x\right), \ldots, \mathrm{j}_{\mathrm{ra}}\left(\right.$ For $\left._{S}, x\right)$ in a different way than described above, eg. as $\operatorname{Mod}_{S}(\{\neg x\})$. If $x \notin A$ we let each $\mathrm{j}_{\mathrm{cwe}}(A, x), \ldots, \mathrm{j}_{\mathrm{ra}}(A, x)$ be $\operatorname{Mod}_{S}(A) \cap \operatorname{Mod}_{S}(\{\neg x\})$. For a formal definition of these contractions see [Giesl, 1992].
} 
The relationship of these functions is illustrated ${ }^{8}$ by figure 4 where "cwe $\rightarrow$ iwe" means that $\mathrm{j}_{\mathrm{cwe}}(A, x) \subseteq \mathrm{j}_{\text {iwe }}(A, x)$ holds for every $A \in \mathcal{T}_{\mathrm{F}}(S)$ and for every $x \in$ For $_{S}$.

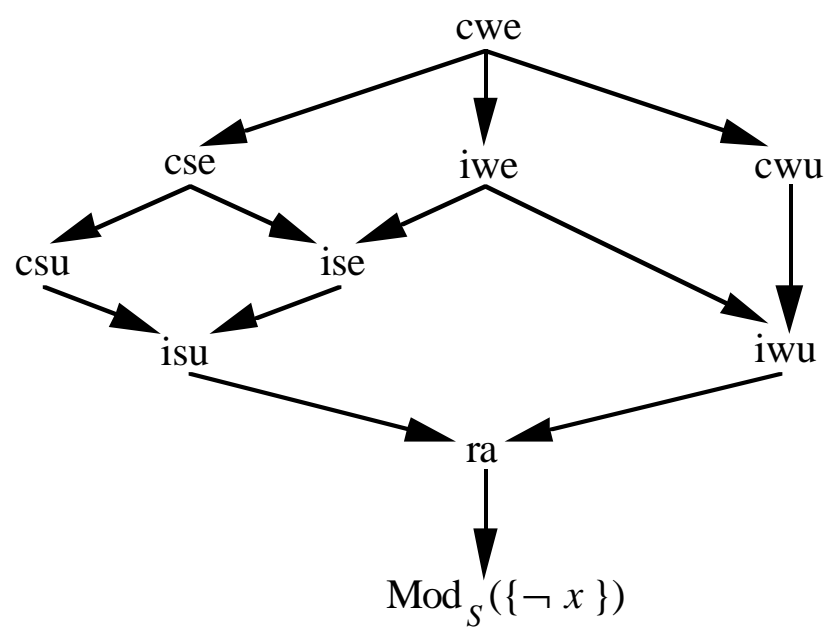

Figure 4: Relationship of the sets of interpretations defining contractions for minimal semantical change

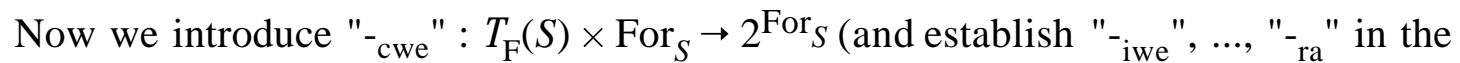
same way).

For every $A \in \mathcal{T}_{\mathrm{F}}(S)$ and for every $x \in$ For $_{S}$ we define

$\operatorname{Mod}_{S}\left(A-{ }_{\text {cwe }} x\right)=\operatorname{Mod}_{S}(A) \cup \mathrm{j}_{\mathrm{cwe}}(A, x)$.

If we choose $r(A, x)$ to be $\operatorname{rat}\left(\operatorname{Mod}_{S}(A)\right) \cup \operatorname{rat}\left(\operatorname{Mod}_{S}(\{x\})\right)$ as long as this union is not empty (and an arbitrary finite non-empty subsignature otherwise) then $\mathrm{j}_{c w e}, \ldots$, $\mathrm{j}_{r a}$ are all reducible to $r(A, x)$. Therefore by theorem 1 we can conclude that "-cwe", ..., "- ${ }^{\text {ra }}$ are rational finite contractions 9 .

All of the semantically defined rational contractions mentioned in the survey of [Katsuno / Mendelzon, 1989] work according to the strategy of minimal semantical change:

[Dalal, 1988], [Satoh, 1988], [Borgida, 1985], [Weber, 1986] and [Winslett, 1987]

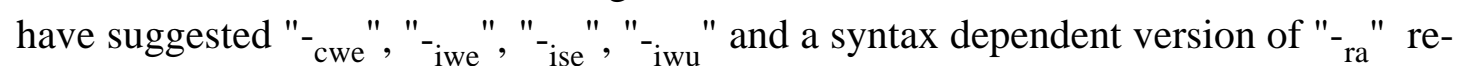
spectively.

Viewed syntactically, their strategy aims at selecting a maximal subsignature $R^{\prime} \subseteq S$ and keeping at least the formulas of $A \cap$ For $_{R^{\prime}}$ in the resulting theory $A-x$. We argue that this is a contraintuitive behaviour because the number of "important" formulas in $A$ concerning a certain $R^{\prime}$ is not equal for different sets $R^{\prime}$ of the same size.

\footnotetext{
${ }^{8}$ If all arrows resulting from the transitivity of $" \rightarrow$ " are added to figure 1 it depicts the complete $" \rightarrow$ " relation on $\{\mathrm{cwe}, \ldots, \mathrm{ra}\}$.

$9 "{ }^{\prime}$ cwe" is even fully rational but the others are not.
} 


\section{Example 4 (cont.)}

We consider clauses ${ }^{10}$ instead of general formulas and regard a clause the more important the less literals it consists of ${ }^{11}$. Then $c \vee d$ is the most important clause of $A$. But $I_{3} \in \operatorname{Mod}_{S}\left(A-_{\mathrm{cwe}} x\right)=\ldots=\operatorname{Mod}_{S}\left(A{ }_{{ }_{\mathrm{ra}}} x\right)$ and $I_{3} \not \# c \vee d$. Therefore $c \vee d \notin A-_{{ }_{\mathrm{cwe}}} x=\ldots=A-_{\mathrm{ra}} x$. So the most important clause of $A$ gets lost in every contraction defined according to the strategy of minimal semantical change.

Therefore one should choose an $R^{\prime}$ such that as many "important" formulas as possible belong to For $_{R^{\prime}}$ and maintain them during contraction. This leads to the strategy of maximal maintenance discussed in the next section.

\subsection{Maximal Maintenance}

As stated in the last section the strategy of minimal semantical change means regarding an interpretation as being "near" to $\mathfrak{I} \subseteq \operatorname{Int}(S)$ if it can be obtained by some "small" change of $\mathfrak{I}$. However in the strategy of maximal maintenance we define an interpretation $I$ to be "nearest" to $\mathfrak{I}$ if the number of "big" changes which result in $I$ when applied to $\mathfrak{I}$ is as large as possible.

By giving the term "big" a precise meaning we now define a concrete contraction according to this strategy. Its aim is to maintain as many important clauses as possible during contraction. We demonstrate that this goal can be reached by extending $\operatorname{Mod}_{S}(A)$ by those $I \in \operatorname{Mod}_{S}(\{\neg x\})$ for which a large number of big ${ }^{12}$ subsignatures $R$ exist such that $I$ can be obtained by generalization of $\operatorname{Mod}_{S}(A)$ with respect to $R$.

Let $\mathfrak{I} \subseteq \operatorname{Int}(S)$ be reducible to a finite subsignature. Then $\mathrm{UG}_{\mathfrak{I}}^{n}(I)$ denotes the number of $\underline{\boldsymbol{u}}$ nuccessful $n$-generalizations of $\mathfrak{I}$ regarding $I$, i.e. the number of all sets $R \subseteq \operatorname{rat}(\mathfrak{I})$ of cardinality $n$ such that $I$ cannot be obtained by generalizing $\mathfrak{I}$ with respect to $R$.

If $\mathfrak{I}=\operatorname{Mod}_{S}(A)$ and if we restrict ourselves to clauses consisting only of relevant atoms of $\mathfrak{I}$ then it can be shown that $\mathrm{UG}_{\mathfrak{I}}^{n}(I)$ is the number of clauses of cardinality $|\operatorname{rat}(\mathfrak{I})|-n$ in $A$ that $I$ evaluates to false.

\footnotetext{
${ }^{10} \mathrm{~A}$ clause is a finite set of literals (propositional variables and their negated counterparts) logically equivalent to the disjunction of its elements.

${ }^{11} \mathrm{We}$ regard statement $a$ as more important than statement $a \vee b$, because the former provides more definite information about the world.

12 with regard to cardinality.
} 
Now we introduce the ordering of successfulness "동 such that $I_{0} \subseteq_{\mathfrak{I}} I_{1}$ iff the number of big changes of $\mathfrak{I}$ by which $I_{1}$ can be obtained is larger (or equal) than the one for $I_{0}$.

\section{Definition 5}

Let $\mathfrak{I} \subseteq \operatorname{Int}(S)$ be reducible to a finite subsignature. Then we define the ordering of successfulness $" \subseteq_{\mathfrak{I}} " \subseteq \operatorname{Int}(S) \times \operatorname{Int}(S)$ for every $\left\{I_{0}, I_{1}\right\} \subseteq \mathfrak{I}(S)$ as

$I_{0} \sqsubseteq_{\mathfrak{I}} I_{1}$

iff

if $\mathrm{UG}_{\mathfrak{I}}^{n}\left(I_{1}\right)>\mathrm{UG}_{\mathfrak{I}}^{n}\left(I_{0}\right)$ then there is a $\mathrm{m}>\mathrm{n}$ such that $\mathrm{UG}_{\mathfrak{I}}^{m}\left(I_{1}\right)<\mathrm{UG}_{\mathfrak{I}}^{m}\left(I_{0}\right)$.

If $\mathfrak{I}=\operatorname{Mod}_{S}(A)$ then this ordering has the effect that $I_{0} \sqsubseteq_{\mathfrak{I}} I_{1}$ holds iff the number of important clauses in $A$ that $I_{1}$ evaluates to true is greater (or equal) than the one for $I_{0}$. So the semantical strategy of preferring interpretations obtainable by as many big changes as possible corresponds to the syntactical goal of maintaining as many important clauses as possible.

For every $A \in \mathcal{T}_{\mathrm{F}}(S)$ and for every $x \in$ For $_{S}$ we let $\mathrm{j}_{\mathrm{cl}}(A, x)$ be the set of all maximal models of $\{\neg x\}$ with respect to ${ }^{-} ﹎{\operatorname{Mod}_{S}(A)}$ ". In fact $\mathrm{j}_{\mathrm{cl}}$ is of a completely different nature than the functions $j$ defined according to the strategy of minimal semantical change. Therefore $\mathrm{j}_{\mathrm{cl}}$ cannot be included in figure $4^{13}$.

Finally we define the clause contraction "- ${ }_{\text {cl }}: \mathcal{T}_{\mathrm{F}}(S) \times$ For $_{S} \rightarrow 2^{\text {For }_{S}}$

for every $A \in \mathcal{T}_{\mathrm{F}}(S)$ and for every $x \in$ For $_{S}$ as

$\operatorname{Mod}_{S}\left(A-_{\mathrm{cl}} \mathrm{x}\right)=\operatorname{Mod}_{S}(A) \cup \mathrm{j}_{\mathrm{cl}}(A, x)$.

\section{Example 4 (cont.)}

Generalization with respect to any three- or four-element subsignature yields $I_{1}, I_{2}$ and $I_{3}$. Therefore $\operatorname{UG}^{4}\left(I_{1}\right)=\operatorname{UG}^{4}\left(I_{2}\right)=\operatorname{UG}^{4}\left(I_{3}\right)=0$ and $\operatorname{UG}^{3}\left(I_{1}\right)=\operatorname{UG}^{3}\left(I_{2}\right)=$ $\mathrm{UG}^{3}\left(I_{3}\right)=0$. For every two-element subsignature generalization yields $I_{1}$ and $I_{2}$, i.e. $\mathrm{UG}^{2}\left(I_{1}\right)=\mathrm{UG}^{2}\left(I_{2}\right)=0$. But $I_{3}$ cannot be obtained by generalization with respect to $\{a, b\}$ as $I_{3}$ is the only interpretation that evaluates a two-element clause of $A$ to

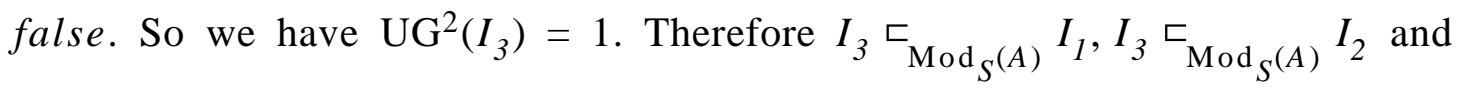
$\mathrm{j}_{\mathrm{cl}}(A, x)=\left\{I_{1}, I_{2}\right\}$. As $\operatorname{Mod}_{S}\left(A{ }_{-\mathrm{cl}} x\right)=\operatorname{Mod}_{S}(A) \cup\left\{I_{1}, I_{2}\right\}$ the most important clause of $A$ is maintained, i.e. $c \vee d \in A-_{\text {cl }} x$.

\footnotetext{
${ }^{13}$ The reason is that there is a theory $A$ and a formula $x$ such that for every function $j$ defined in section $3.1 \mathrm{j}_{\mathrm{cl}}(A, x) \cap j(A, x)=\varnothing$ holds although $x$ is not a tautology.
} 
Again $\mathrm{j}_{\mathrm{cl}}(A, x)$ is reducible to $r(A, x)$ as chosen in section 3.1. Therefore by theorem 1 "-cl" is a rational finite contraction. Actually "-cl" has the advantage of being even fully rational while the contractions of the last section (with the exception of "- cwe") are not.

But the main reason for preferring the clause contraction to the ones of minimal semantical change is that it is defined according to the strategy of maintaing a maximal set of important formulas.

\section{Conclusion}

We have presented semantical characterizations of rational contractions and in particular of finite rational contractions, i.e. the subclass of functions transforming finitely representable theories into such theories again. Due to this result it is now possible to understand the nature of these functions and to define and examine concrete contractions usable in practical applications.

When examining the previously known rational contractions by means of this characterization we have discovered that they all follow a strategy of minimal semantical change. As this strategy does not have the desired syntactical property we have introduced the new strategy of maximal maintenance which has led to the definition of clause contraction.

\section{References}

[Alchourron / Makinson, 1982] C. E. Alchourron and D. Makinson, On the Logic of Theory Change: Contraction Functions and their associated Revision Functions, Theoria 48 , pp. $14-37$

[Alchourron / Gärdenfors / Makinson, 1985] C. E. Alchourron, P. Gärdenfors and D. Makinson, On the Logic of Theory Change: Partial Meet Contraction and Revision Functions, Journal of Symbolic Logic 50, pp. 510-530

[Borgida, 1985] A.Borgida, Language Features for Flexible Handling of Exceptions in Information Systems, ACM Transactions on Database Systems 10, pp. 565-603

[Dalal, 1988] M. Dalal, Investigations into a Theory of Knowledge Base Revision: Preliminary Report, Proceedings of the Seventh National Conference on Artificial Intelligence, Saint Paul, Minn., pp. 475-479 
[Gärdenfors, 1988] P. Gärdenfors, Knowledge in Flux - Modeling the Dynamics of Epistemic States, MIT Press

[Giesl, 1992] J. Giesl, Die Semantik rationaler Kontraktionsfunktionen, Diploma Thesis, Department of Computer Science, University of Karlsruhe

[Giesl / Neumann, 1993] J. Giesl and I. Neumann, The Semantics of Rational Contractions (Poster Abstract), Proceedings of the 6th Portuguese Conference on AI, EPIA'93, Porto, Portugal

[Giesl / Neumann, 1994] J. Giesl and I. Neumann, Strategies for Semantical Contractions (Poster Abstract), Proceedings of the 18. Deutsche Jahrestagung für Künstliche Intelligenz, KI-94, Saarbrücken, Germany

[Grove, 1988] A. Grove, Two Modellings for Theory Change, Journal of Philosophical Logic 17, pp. 157-170

[Katsuno / Mendelzon, 1989] H. Katsuno and A. O. Mendelzon, A Unified View of Propositional Knowledge Base Updates, Proceedings of the 11th International Joint Conference on Artificial Intelligence, Detroit, Mich., pp. 1413-1419

[Satoh, 1988] K. Satoh, Nonmonotonic Reasoning by Minimal Belief Revision, Proceedings of the International Conference on Fifth Generation Computer Systems, Tokyo, Japan, pp. 455-462

[Weber, 1986] A. Weber, Updating Propositional Formulas, Proceedings of the First Conference on Expert Database Systems, pp. 487-500

[Winslett, 1987] M. Winslett, Updating Databases with Incomplete Information, Technical Report STAN-CS87-1143, Stanford University 